\title{
P I 4-09. Use of HIV rapid tests for assessment of persistence of vaccine induced antibodies among HIV vaccine recipients
} E Karita*1, K Kayitenkore ${ }^{2}$, R Bayingana ${ }^{1}$, F Sebahungu ${ }^{1}$, J Bizimana ${ }^{1}$, K Grabowski ${ }^{1}$, A Tichacek ${ }^{3}$, C Schmidt ${ }^{4}$, P Fast ${ }^{4}$, E Hunter ${ }^{5}$ and S Allen ${ }^{3}$

Address: ${ }^{1}$ Projet San Francisco, Kigali, Rwanda, ${ }^{2}$ World Health Organization, Geneva, Switzerland, ${ }^{3}$ Emory University, Rollins School of Public Health, Atlanta, GA, USA, ${ }^{4}$ International AIDS Vaccine Initiative, New York, USA and ${ }^{5}$ Emory University, Emory Vaccine Center, Atlanta, GA, USA

* Corresponding author

from AIDS Vaccine 2009

Paris, France. 19-22 October 2009

Published: 22 October 2009

Retrovirology 2009, 6(Suppl 3):PI97 doi:10.1 186/1742-4690-6-S3-PI97

This abstract is available from: http://www.retrovirology.com/content/6/S3/PI97

(c) 2009 Karita et al; licensee BioMed Central Ltd.

\section{Background}

Induction and persistence of vaccine induced antibodies among recipients of HIV vaccines may pose a diagnostic challenge. We investigated the performance and usefulness of rapid tests for the detection of HIV antibodies among healthy HIV-uninfected people previously enrolled in a phase I HIV vaccine trial in Kigali, Rwanda.

\section{Methods}

Between September 2005 and May 2006, 57 healthy, HIVuninfected adult volunteers ( 36 males and 21 females) were enrolled in a phase-I, randomized, placebo-controlled HIV vaccine trial at Projet San Francisco in Kigali, Rwanda. At the end of the trial and after unblinding, all volunteers were asked to participate in a long-term followup study for continuous clinical and immunological evaluation. HIV testing using three rapid tests (Abbott Determine $^{\mathrm{TM}}$ HIV1-2, Trinity Biotech Capillus ${ }^{\mathrm{TM}}$ HIV-1/HIV-2 and Trinity Biotech Uni-GoldTM HIV) and an ELISA test (Bio-Mérieux Vironostika HIV Uniform II Ag/Ab) was performed every three months.

\section{Results}

Of the 57 volunteers enrolled in the trial, 13 received placebo, 13 received one dose of a recombinant HIV-1 adenoviral type 5 vector vaccine ( $\mathrm{Add} 5)$, and 31 received 3 doses of an HIV-1 DNA plasmid vaccine followed by one dose of rAd5. Of these 44 vaccine recipients, 41 produced HIV antibodies during the trial period. After 18 to 24 months of follow-up, 32 volunteers still test HIV-positive on the ELISA test, but only one volunteer tested persistently HIV positive using HIV rapid tests.

\section{Conclusion}

Most vaccine recipients produced HIV antibodies that have persisted for at least two years. These vaccineinduced antibodies are predominantly detected by a commercial ELISA test, and not by the three HIV rapid tests that were evaluated. This study suggests that HIV rapid tests can be very useful in the long-term follow-up of HIV vaccine recipients. 